\title{
Investigation of contact edge effects in the channel of planar Gunn diodes
}

\author{
A Mindil, G M Dunn, A Khalid, and C H Oxley
}

\begin{abstract}
The effect of the edge of the channel on the operation of Planar Gunn diodes has been examined using Monte Carlo simulations. High fields at the corner of the anode contact are known to cause impact ionization and consequent electroluminescence, but our simulations show that the Gunn domains are attracted to these corners, perturbing the formation of the domains which can lead to chaotic dynamics within the rest of the channel leading to uneven heating and reduced RF output power. We show how novel shaping of the electrical contacts at the ends of the channel reduces the attraction and restores the domain wave-fronts for good device operation.
\end{abstract}

Index Terms - Planar Gunn diode, Monte Carlo, GaAs, Channel Edge

Manuscript received July 12, 2019. This work was supported by the Ministry of Higher Education of Saudi Arabia.

A. Mindil is with the Department of Physics, School of Natural and Computing Sciences, University of Aberdeen, Aberdeen AB24 3UE, U.K. (e-mail: r01amhm@abdn.ac.uk).

G. Dunn is with the Department of Physics, School of Natural and Computing Sciences, University of Aberdeen, Aberdeen AB24 3UE, U.K. (e-mail: g.m.dunn@abdn.ac.uk).

A. Khalid with the Centre of Electronic Warfare, Information and Cyber, Cranfield University, Defence Academy of the United Kingdom, Shrivenham SN6 8LA, U.K. (e-mail:,Ata.khalid@cranfield.ac.uk) U.K

C. H. Oxley was with De Montfort University, Leicester LE1 9BH,

\section{INTRODUCTION}

lanar Gunn devices have been suggested as an alternative to the vertical Gunn's because of the ease with which they can be integrated into a monolithic microwave integrated circuits (MMIC) [1]-[5]. Furthermore, higher frequencies are potentially possible in planar Gunn diodes and operation in fundamental mode up to $164 \mathrm{GHz}$ [2] have been demonstrated and yet higher frequencies would seem to be possible [6].

Power from these devices however has not yet reached its full potential as suggested by both simulation and an experimental investigation of the current distribution within the channel of the devices using a micro-particle IR thermal measurement technique [7]. The measured temperature profiles were found to be asymmetrical implying significantly lower current densities towards the edge of the channel. It was proposed that this difference in current density across the channel of the device may be one of the causes of lower than expected RF output power observed with current planar Gunn diode structures. Further, it was suggested that the shape of the electrical contact at the edge of the device might be responsible and that shaping of the electrical contact at the channel edge might mitigate the uneven current along the channel.

Earlier investigations of impact ionization in Planar Gunn structures have shown high electro luminesce (EL) along the length of the anode contact with a higher level of EL at both ends of the channel [8]. This suggests high electric fields exist, particularly where we might expect such fields, at the sharp corner of the electrical contacts. In a previous study [9] it was found that geometrical modifications to the anode contact along its length could reduce the effect along its length, but had little effect on the high electric fields at the corners of the anode contact.

In this present work, using Monte Carlo simulations, we will look at the effect of the corner of the anode contact on the progress of Gunn domains and at the effect of shaping the contacts to restore consistent domain wave-front across the width of the channel from cathode to anode.

\section{Device Simulation}

An ensemble Monte Carlo method was used to simulate the carrier transport in the studied devices with an established 2-D Ensemble Monte Carlo (EMC) transport model, details of 
which are given in [10], which has been validated against many similar experimentally realized devices [1-10]. As in [11] a doping notch next to the cathode contact was used to simulate the effect of the cathode contact to precipitate domain formation. A typical mesh of 100 by 100 were used with 100,000 super particles giving a weight of the order of one super particle per electron in this structure. All electrons were weighted equally and a typical run time was about 6 hours to simulate 50ps. A field adjusting time step of $1 \mathrm{fs}$ was used and the boundary conditions used are the usual Dirichlet conditions at the contacts with defined potentials and Neumann conditions at other boundaries assuming continuity of the electric field. The simulations were considered valid provided that the domain (and electric field) were normal to the lower boundary, going down along the un-simulated part of the channel).

The gallium arsenide (GaAs) planar Gunn diode has been described in detail in [1]-[8] and the electrode geometry is shown in fig 1.

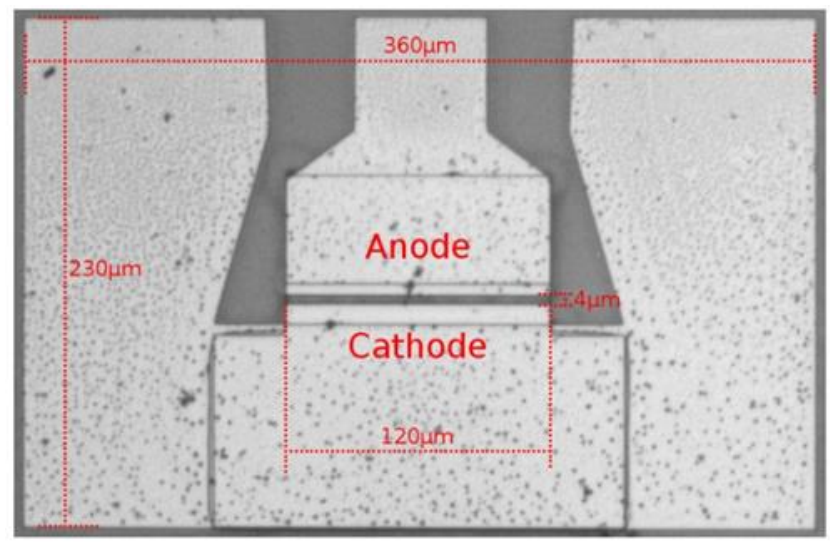

Fig. 1. Planar Gunn Diode with a 4x120 $\mu \mathrm{m}$ channel [10].

For ease and accuracy of simulation, we only simulated the top $7.0 \mu \mathrm{m}$ of the channel length to include the sharp edges of the anode and cathode contacts, see Fig 2(a). The width of the channel was $1.3 \mu \mathrm{m}$. Some longer device simulations on devices up to $12 \mu \mathrm{m}$ were performed to confirm the validity of the boundary conditions and verify that the simulation of the top $7.0 \mu \mathrm{m}$ was the same as the simulations where only $7.0 \mu \mathrm{m}$ was simulated.

It was argued in [7] that the reason for the non-uniform temperature variation across the channel was uneven heating due to poor operation at the channel edge in this un-optimized device, and that should good operation exist in an optimized device, the heating would therefore be more even. Our simulations were therefore done on the assumption of good operation in an optimized device with a uniform lattice temperature of $300 \mathrm{~K}$ and at a bias of $1.36 \mathrm{~V}$. The effect of any temperature variation, which might exist for any other reason, though out of the scope of this investigation, might also have some impact on the uniformity of the motion of the Gunn domain as there is some temperature dependence on the velocity of a domain and is another possible source of less than optimal performance of these planar devices. However frequency stability with respect to temperature is known to be extremely good in GaAs [12] and figure 2 shows the simulated variation in transit period for this size of device as a function of temperature over several hundred Kelvin amounts to a change of only $0.008 \mathrm{ps} \mathrm{K}^{-1}$ and as such is not expected to have a significant impact on the device operation for variations even of the order of $10 \mathrm{~K}$ as observed in the nonoptimized device in [7].

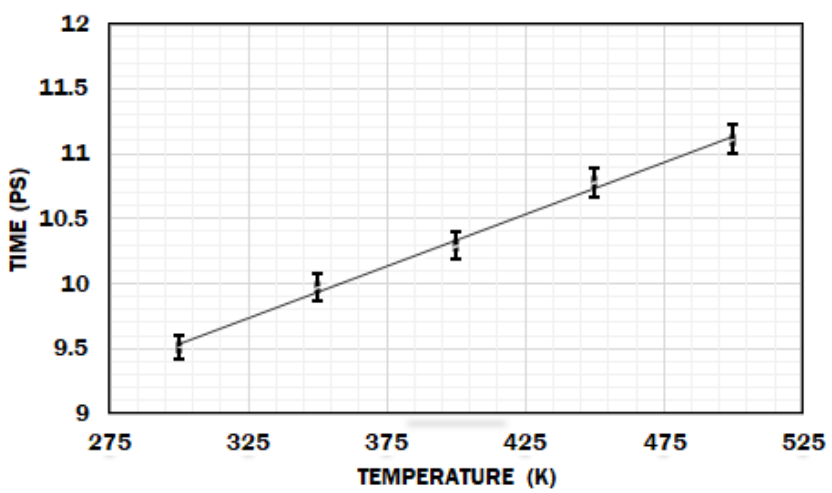

Fig 2. The effect of temperature on the period of transit of a Gunn domain in the planer structure.

Some simulations with RF feedback were done, though the device in [8] was not in a cavity and we therefore concentrated on a constant applied voltage.

To achieve the purpose of this study, we simulated the normal sharp corner device structure (figure 3a) and also explored a large number of different variations to the shape of the edge of the channel which fall into four categories, convex-convex or blunt contacts (figure $3 \mathrm{~b}$ ), convex-concave (figure $3 \mathrm{c}$ ), concave-convex (figure 3d) and concave-concave (figure $3 \mathrm{e}$ ). Each were varied in the size of curvature (in the width across the channel and length along the channel).

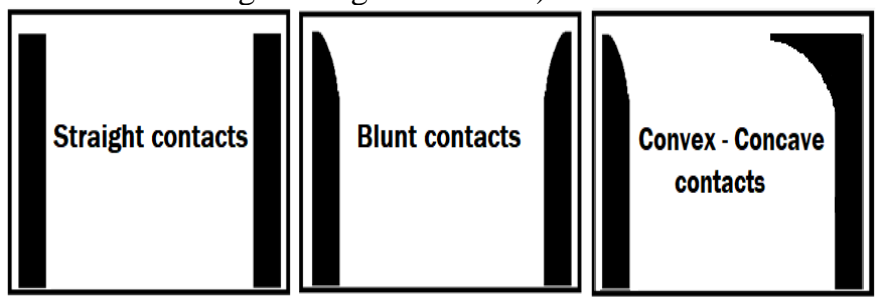

(a)

(b)

(c)

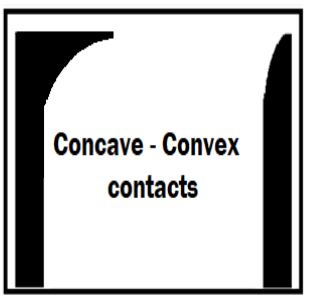

(d)

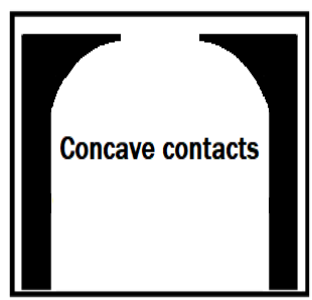

(e)
Fig. 3. (a) Schematic of the straight sharp corner contacts, (b) blunt corner contacts, (c) convex-concave corner contacts. (d) concave-convex corner contacts. (e) concave -concave corner contacts.

\section{RESULT AND DISCUSSIONS}

Figure 4 shows the domain structure in the $12 \mu \mathrm{m}$ planar 
Gunn diode with sharp contact edges (Figure 3a) after 5 transits of the domain. Rather than being well formed, straight and parallel to the electrical contacts it is observed that the high electric field at the edge acts to attract the domain, to some extent anchoring it. The domain is stretched out and weakened and this distortion becomes progressively worse and more extensive down the length of the channel with each subsequent transit. The effect is reduced but still very noticeable when there is a RF feedback potential at the natural frequency of the device.

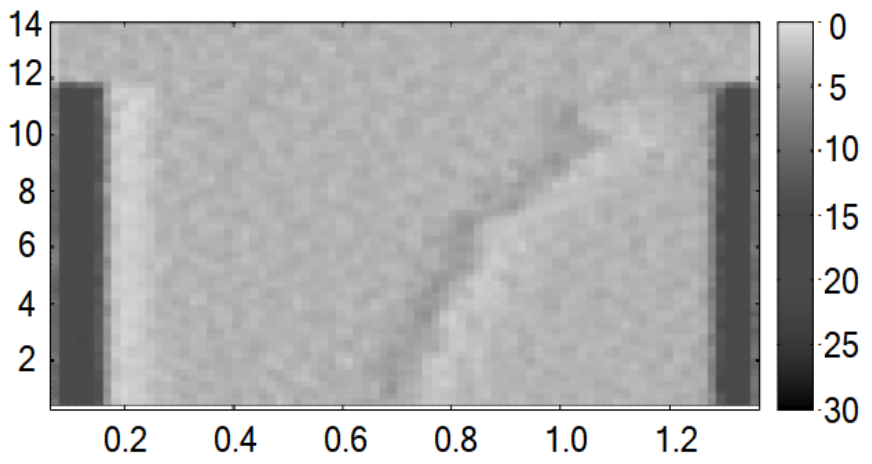

Fig. 4. The electric field at $110 \mathrm{ps}$ for $12.0 \mu \mathrm{m}$ length and $1.3 \mu \mathrm{m}$ width. The device under the effect of $1.36 \mathrm{~V}$ bias during the transit region.

Figures 4 a (I-III) \& b (I-III) show the simulated electric field during the transit of a Gunn domain in the top $7.0 \mu \mathrm{m}$ of the channel for the sharp (Figure 4a) and blunt (Figure 4b) corners at (I)79.9, (II)82.2, and (III)84.6 ps respectively for both devices (after several 9 ps transits from the start of the simulation).

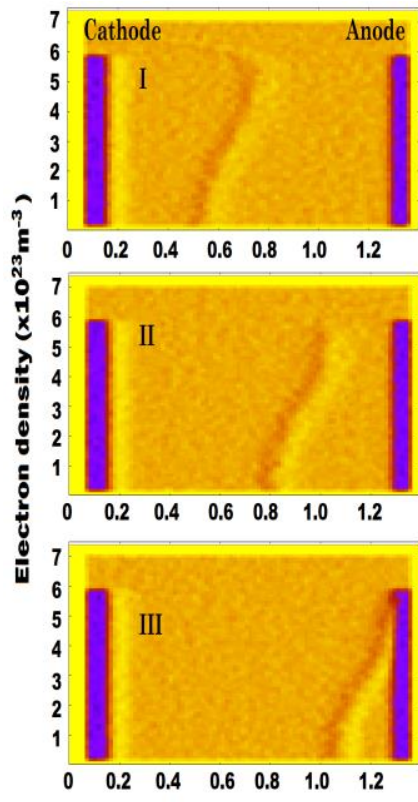

(a)

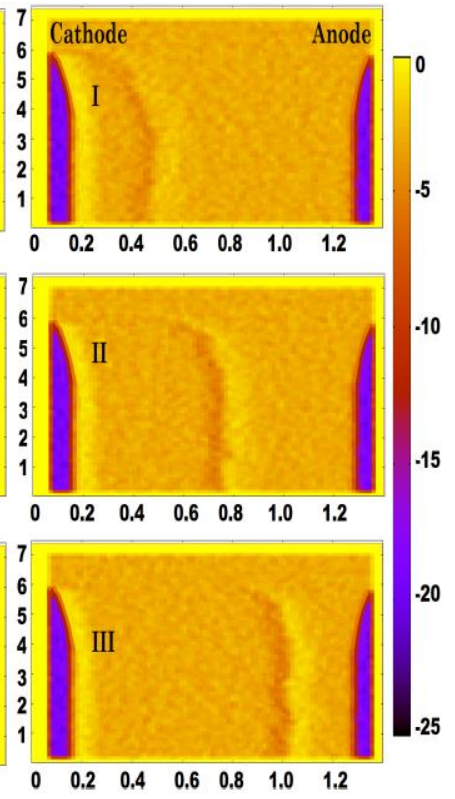

(b)
Fig. 5. (I), (II), and (III), shows the electron density (in units of $10^{23} \mathrm{~m}^{-3}$ ) a $1.3 \mu \mathrm{m}$-width device under the effect of $1.36 \mathrm{~V}$ bias at several instants during the transit in the straight device (a) and blunt or convex-convex device (b). In both devices (I) The domain forms at $79.9 \mathrm{ps}$ from the cathode. (II) $2.4 \mathrm{ps}$ later the domain has progressed further towards the anode. (III) after another $2.4 \mathrm{ps}$.
The distortion is very noticeable in (a), but apart from the top in (b) the domain is straight and parallel with the anode.

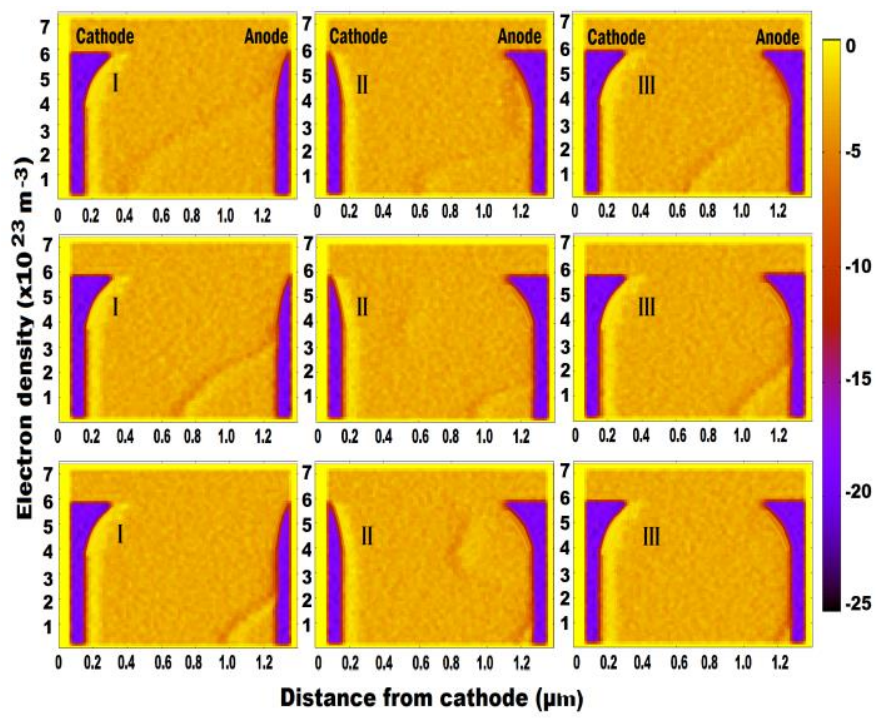

(a)

(b)

(c)

Fig. 6. (I), (II), and (III), shows the electron density (in units of $10^{23} \mathrm{~m}^{-3}$ ) a $1.3 \mu \mathrm{m}$-width device under the effect of $1.36 \mathrm{~V}$ bias at several instants during the transit in the convex-concave device (a), concave-convex device (b) and concave -concave device. In each device (I) The domain forms at $76.1 \mathrm{ps}$ from the cathode. (II) $2.4 \mathrm{ps}$ later the domain has progressed further towards the anode. (III) after another 2.4 ps. The distortion is remarkable.

In (Figure 4a), the domain forms non-uniformly along the channel at the cathode contact corner first and then transits to the anode contact with the domain in the rest of the channel trailing behind the domain at the top. The domain is then attracted to the anode contact corner and reforms at the cathode contact top corner before the rest of the domain has reached the anode contact. The kink in the domain becomes more marked, stretched and weakened and spreads further down the channel with each subsequent transit, significantly distorting its wave-front. The effect is reduced but still very noticeable when there is a RF feedback potential at the natural frequency of the device.

Various attempts were made to reduce this effect by shaping of the edge of the contact. Initially we favoured the concaveconcave shaping as we believed that the problem may be due to the spreading and weakening of the field at the edge of the channel. However, this proved not to be the case. The domains instead were attracted even more to the sharp corner of the anode contact and the resulting distortion of the domain was as bad or worse than for the straight contact. Figure $6 \mathrm{c}$ shows the domain in transit in this structure and it is clearly seen to be more distorted and weakened than the domain in figure $5 \mathrm{a}$ at similar times. The domains shape was also greatly influenced by the shape of the cathode contact and attempts to compensate by using combinations of convex anode, retaining the concave cathode also failed (see figure 6b). Here it can be seen that the domain has been so weakened that it has broken up into several incoherent fragments. We also tried a combination concave cathode and convex anode (figure 6a) however the domain here can also be seen here to be more distorted than that in figure 5a. 
The convex-convex combination, however, did show immediate improvements over the straight contact and by increasing the length of the blunt shape to 2 microns along the length of the channel and 0.1 microns along the width the problem is resolved as shown (Figures 5b). The domain forms at the cathode contact, taking on the shape of the contact. The electric field is weaker at the top of the device so the domain moves a little faster here than the rest of the domain (because of negative differential resistivity) catching up with the rest of the domain during the transit until the domain is almost straight by the time of reaching the anode contact. The domain is less attracted to the blunt corner, flipping over to take the shape of the anode contact only briefly before reforming at the cathode. The domain wave-front is consistently parallel with the contacts.

Figures 7 (a, b \& c) shows the current for the sharp corner, an intermediate design and the optimal blunt corner contact respectively. The sharp corner contact current waveform shows broader and thicker peaks (full width at half maximum of 3 ps and increasing) because the domain washes up against

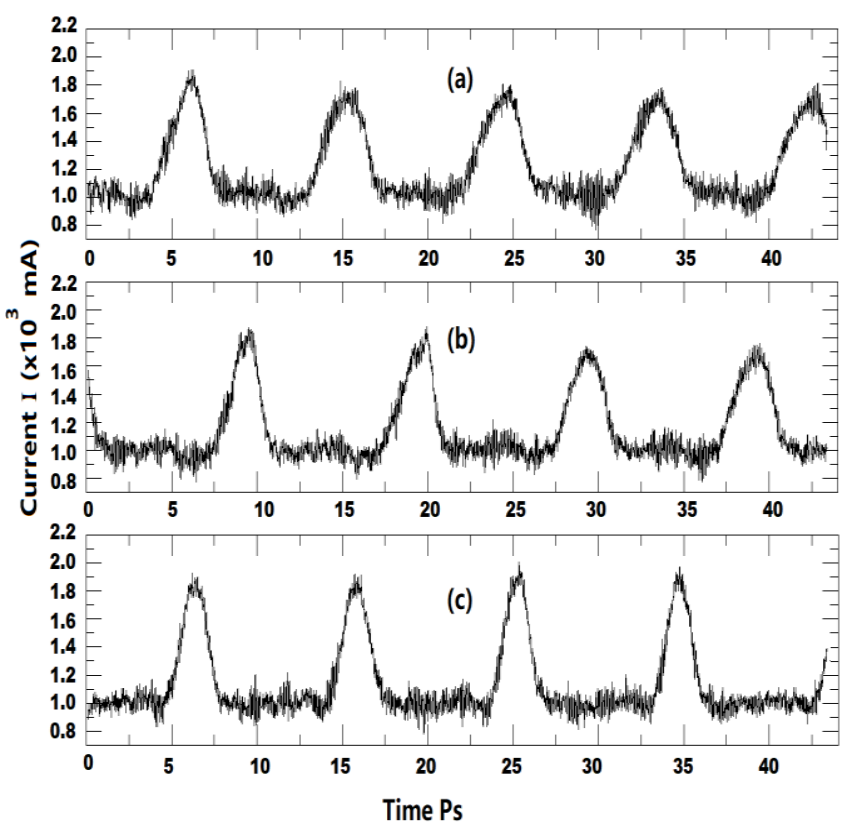

Fig. 7. Total current from Monte Carlo simulation due to different channel contacts devices for width $1.3-\mu \mathrm{m}$. (a) Current for straight contacts shape. (b) Current for intermediate design (between the straight and blunt shapes) contacts planar Gunn device. (c) Current for blunt contacts planar Gunn device.

the anode gradually, hitting the top first and then down the length of the channel. The exact shape of the pulse is therefore a function of the channel length and also time, because the waveform deteriorates, extending further down the channel with each subsequent transit, so the peaks become broader in time. In a cavity, with RF feedback, the phase lag in the wavefront could give a negative feedback with consequences of lowering the possible power output of the device. On the other hand, the current in optimum blunt shape contacts (Figure 7c) is sharper (fwhm 2ps) and more precise because the domain hits the whole length of the anode at the same time and so is not a function of the channel length and does not deteriorate with time. Figure $7 \mathrm{~b}$ shows the current for a contact which changes by 1 micron along the length of the channel by 0.1 microns along the width - intermediate between the optimum blunt and sharp contacts. The current wave form shows a broadening over the optimum contact shape ( 2 microns by 0.1 microns) though is better than the straight contact.

\section{CONCLUSION}

The cause of uneven heating within the channels of planar Gunn diodes has been investigated. The attraction of domains to the sharp corner of the anode has been shown to lead to a kink developing in the domain which can then spread down the length of the channel leading to chaotic and weakened Gunn domains near the channel edges.

A comparison has been made between sharp cornered contacts and novel blunted corner contacts. In the blunt design, the domains moved symmetrically through transit region along the length of the channel device. The resulting current was consequently considerably more ordered with sharper and improved defined peaks.

In conclusion, a new shape of contacts for planar Gunn diode have been presented which eliminates the distortion of the domain associated with the sharp corners of the traditional design and that this design leads to well-ordered domains which will have improved RF power output and device performance.

\section{ACKNOWLEDGMENT}

We thank the University of Aberdeen for providing the necessary support and The Ministry of Higher Education of Saudi Arabia for their financial support.

\section{REFERENCES}

[1]. A. Khalid, N. J. Pilgrim, G. M. Dunn, M. C. Holland, C. R. Stanley, I. G. Thayne, and D. R. S. Cumming, "A planar Gunn diode operating above 100 GHz,” IEEE Electron Device Lett., vol. 28, no. 10, pp. 849- 851, Oct. 2007.

[2]. Khalid, A. et al. (2013) In0.53Ga0.47As planar Gunn diodes operating at a fundamental frequency of $164 \mathrm{GHz}$. IEEE Electron Device Letters, 34(1), pp. 39-41. (doi:10.1109/LED.2012.2224841)

[3]. Khalid, A. et al. (2014) Terahertz oscillations in an In0.53Ga0.47As submicron planar gunn diode. Journal of Applied Physics, 115(11), p. 114502. (doi:10.1063/1.4868705)

[4] N. J. Pilgrim, A. Khalid, G. M. Dunn, and D. R. S. Cumming, "Gunn oscillations in planar heterostructure diodes," Semicond. Sci. Technol., vol. 23, no. 7, pp. 075 013-1-075 013-10, Jul. 2008.

[5] C. Li, A. Khalid, S. H. Paluchowski Caldwell, N. J. Pilgrim, M. C. Holland, G. M. Dunn, and D. R. S. Cumming, "Enhancement of power and frequency in planar Gunn diodes by introducing extra deltadoping layers," Microw. Opt. Technol. Lett., vol. 53, no. 7, pp. 1624-1626, Jul. 2011.

[6] A. Khalid, G. M. Dunn, R. F. Macpherson, S. Thoms, D. Macintyre, C. Li, M. J. Steer, V. Papageorgiou, I. G. Thayne, M. Kuball, C. H. Oxley, M. 
Montes Bajo, A. Stephen, J. Glover, and D. R. S. Cumming, "Terahertz oscillations in an In0.53Ga0.47As submicron planar Gunn diode," J. Appl. Phys., vol. 115, no. 11, p. 114502, mar 2014. [Online]. Available: http://aip.scitation.org/doi/10.1063/1.4868705 doi: 10.1063/1.4868705

[7] Glover, J., Khalid, A. , Cumming, D., Dunn, G. M., Kuball, M., Bajo, M. M. and Oxley, C. H. (2017) Thermal profiles within the channel of planar gunn diodes using micro-particle sensors. IEEE Electron Device Letters, 38(9), pp. 1325-1327. (doi:10.1109/LED.2017.2731961)

[8] M. Montes, G. Dunn, A. Stephen, Ata Khalid, C. Li, D. Cumming, C. H. Oxley, R. H. Hopper, and M. Kuball, IEEE Trans. Electron Devices 59, 654 (2012).

[9] Montes Bajo, M., Dunn, G., Stephen, A., Khalid, A., Cumming, D. R. S., Oxley, C. H., Glover, J. \& Kuball, M. 28 Mar 2013In : Journal of Physics D: Applied Physics. 113, 12, 6 p., 124505

[10] Pilgrim N.J., Khalid A., Dunn G.M. and Cumming D.R.S., "Gunn oscillations in planar hetero structure diodes", Semiconductor Science and Technology, (2008), 23, 075013.

[11] Pilgrim, N J, Khalid A., Dunn G. M. and Cumming D.R.S., "Contact Shaping in planar Gunn diodes", Phys. Status Solidi C 8, (2011), DOI 10.1002/pssc.201000539.

[12] N Priestley and N. Farrington, "Millimetere-Wave Gunn Diode Technology and applications" ARMMS RF

Microw.Soc. (2010), pp 1-10. 